\title{
Urgences
}

\section{Comment nous retrouverons-nous...}

\section{Alain St-Yves}

Numéro 3, 4e trimestre 1981

URI : https://id.erudit.org/iderudit/025042ar

DOI : https://doi.org/10.7202/025042ar

Aller au sommaire du numéro

Éditeur(s)

Urgences

ISSN

0226-9554 (imprimé)

1927-3924 (numérique)

Découvrir la revue

Citer ce document

St-Yves, A. (1981). Comment nous retrouverons-nous... Urgences, (3), 33-39.

https://doi.org/10.7202/025042ar

Ce document est protégé par la loi sur le droit d'auteur. L'utilisation des services d'Érudit (y compris la reproduction) est assujettie à sa politique d'utilisation que vous pouvez consulter en ligne.

https://apropos.erudit.org/fr/usagers/politique-dutilisation/
Cet article est diffusé et préservé par Érudit.

Érudit est un consortium interuniversitaire sans but lucratif composé de l'Université de Montréal, l'Université Laval et l'Université du Québec à Montréal. Il a pour mission la promotion et la valorisation de la recherche. https://www.erudit.org/fr/ 


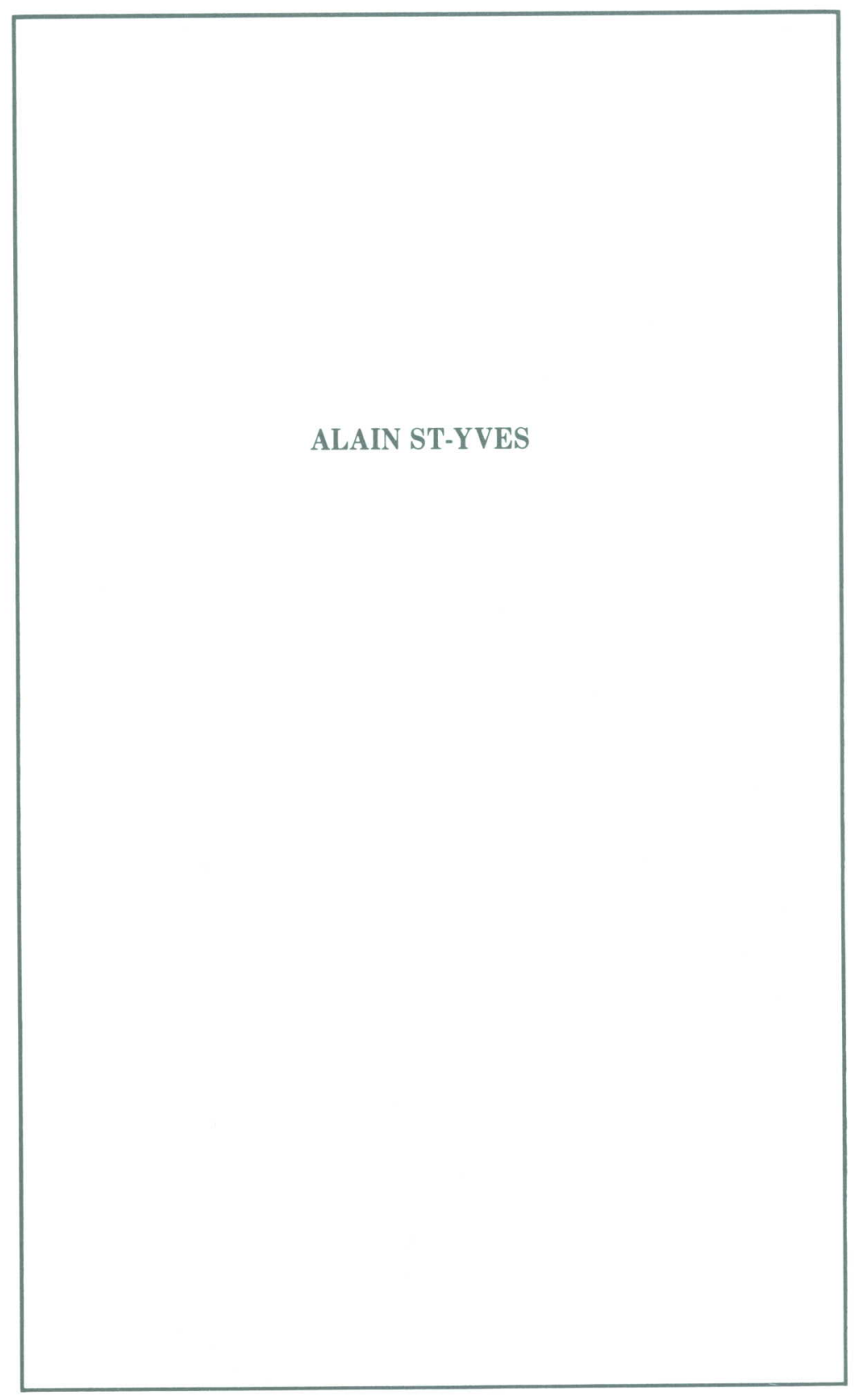


Comment nous retrouverons-nous toujours comptant sur le hasard qui se fait tard qui se fait loin et sur les déroutes qui mènent parfois au seuil de nos portes mal fermées

et de nos fenêtres fragiles qui tremblent

comment referons-nous encore une fois

nos dos un peu moins courbés et nos têtes un peu plus hautes un peu plus follement belles

comment trouver les lumières dignes

de nos plus longues nuits à ramer à contre-courant

en ces petits matins qui brillent pâlottement

où la tendresse vient boire en nos os

et se refaire le visage et le fleuve

elle la toujours traquée d'éternelles inquiétudes

comment porterons-nous encore

la charge de nos intimités de nos émotivités

hors des secrets et hors des doutes

avec le poids de la découverte d'oasis plus fortes que le rêve plus loin que les mirages qu'on s'invente pour détourner la peur nous les extrêmes séparés

batailleurs de l'infini en nos têtes ajourées

laissant passer toujours un peu de soleil de lune et de planète

à qui raconterons-nous la nuit venue à pas de loup

ô belle nuit qui nous love d'étoiles à pas très doux

si ce n'est à nous-mêmes les don Quichotte du radeau de la 
combien d'espaces parallèles traverserons-nous avant que de nous retrouver nous redonner surpris heureux et fous dans nos bras débraillés nous serrant nous captant

à l'abri des griffes des radoteuses règles qui nous font les jeux pervers quand nos yeux ne sont que regards d'Amour

comment éviterons-nous le mensonge et même le songe qui nous trompe pour ne pas rester trop longtemps blessés sachant l'éphémère du voyage qui prend l'eau qui prend le vent et les tourments

où irons-nous un peu nous reposer des noires nouvelles des longs deuils et de la réalité qui craque et craque et craque et nous fait nous refermer comme une cage

où irons-nous nous reposer un peu du monde qui n'en finit plus de tourner en maux de coeur et en terribles nausées

où irons-nous refaire la marée nous abeaudir et cacher le peu qui nous colle à la vie n'ayons plus honte et de nos cris et de nos larmes et de nos peurs de nos gestes maladroits et de nos coeurs peuplés de fantômes allons rapproche-toi de moi par le dedans continuons la route très lentement

Comment nous retrouverons-nous comptant sur le hasard pour déjouer le destin par des cailloux laissés en nos amours ô mon Amour

Comment nous retrouverons-nous toujours

comptant sur le hasard qui se fait tard qui se fait loin

comment continuer la révolte contre les mécréants terrestres qui font chavirer la vie dans leur manière de voir la vie et de la tenir sous leur pouvoir ô faiseurs de destin grimaçant que seule peut contredire la mort 
comment être du même combat avec nos poings complices sans y laisser des trous en nos veines en nos ventres où les rivières coulent et se croisent pour aller prendre le large en étant poussées par le coeur fraternelle force

comment sortirons-nous contents de nos usines à vapeur où le bruit devra devenir un chant $\mathrm{d}^{\prime}$ oiseau murmure de feuilles si nous voulons que nos oreilles demeurent humaines

comment irons-nous mourir tranquillement dans l'espérance en paix avec l'universel et toutes les galaxies heureux d'avoir été ce passant qui a semé des fleurs et des forêts et des enfants fiers d'avoir été le passant le trépassant qui recommence le courage et reprend le travail en d'autres terres

comment serons-nous ce courage qui grandira la vie jusqu'à la rendre plus près de Dieu jusqu'à le dépasser pour le faire lui-même s'agrandir

ô plus grandement que toute éternité

où trouverons-nous la force de donner à la vérité toute la vie qu'elle doit avoir pour être l'unique lumière qui nous replacera dans ce qu'on appelle bonheur mais qui est plus mais qui est tant tellement plus fort tellement plus loin

et bien plus haut que peuvent aller nos espérances allons rapproche-toi de moi par le dedans soyons complices du même vent qui nous habite

comment nous retrouverons-nous intérieurement comptant sur le hasard pour déjouer le destin avec des plumes laissées en nos amours ô mon Amour 


\section{à Richard}

Moi je n'avais rien à dire

il nous arrive parfois de n'avoir rien à dire

je n'avais rien à dire et pas plus à redire

je n'étais pas en état de maudire

pas en état de radoter de chatouner de gueuler de pleurer

de minoucher de placoter de crier de murmurer de chanter

de vilipender de rechigner de chuchoter d'avouer

de dériver comme un poème d'outre-mots

à la recherche d'une page où s'étendre et tendre les lignes

J'étais comme une eau

qui n'attend ni baigneur ni bateau

et qui par un temps où la mélancolie joue de l'équilibre

n'a que des culs de canards et d'oies blanches

comme unique firmament qui le rattache à la vie

Moi j'étais comme une mer jetée dans une bouteille je $\mathrm{n}^{\prime}$ avais rien à dire rien à délirer

mais j'étais pourtant bien rempli

un bouchon dans chaque oeil

empêchait la réalité de trop sortir de moi de mon chef-lieu

et de filer comme une ombre dans la nuit ajourée d'étoiles

Rien n'était pourtant à noyer

malgré les ballotements en dehors de moi comme en dedans

rien n'était à noyer rien à boire

était-ce bon ou bien ou finement fin

et trop subtil pour les mots

mais je parle déjà trop

moi qui crois n'avoir rien à dire

parce qu'il arrive à tout un chacun de n'avoir rien à dire 
parce qu'il arrive au vent de ne pas tempêter ou rafraîchir et d'être incognito dans la température

Comment convaincre ma parole du bien-être qu'on retire à se taire à se terrer parfois tranquillement tranquille

Je pourrais vous parler de mon Amour

de mon bel Amour tellement unique qui a vingt prénoms et plus vous dire qu'elle est brune avec un peu de roux ou tout à fait blonde qu'elle a de longs cheveux qui courrent comme des vagues vers les plages de ses reins où il fait bon mettre les freins lentement avec les mains qu'elle a bouche grande et des jambes qui mènent à Rome qu'elle a des seins petits ou plus gros ou pointus ou bien ronds tout dépendant des nuits je pourrais vous la décrire de mille et une manières et ne jamais me tromper vous la faire plus ronde ou plus longue vous parler des cachettes en elle de la nuque et du beau cou et de beaucoup de regards des petits pieds qui font de grands pas dans le courage quotidien des hanches qui changent au détour des lumières ô mon bel Amour unique et mutiple ô mon bel Amour

Je pourrais dire des vérités qui n'ont pas à faire de galipettes à baguenauder vous dire que contrairement à ce que l'on prétend ce qu'on ne sait pas fait toujours mal vous dire mon dédain des guerres et des gens qui les fabriquent qui jouent avec leur pouvoir horrifiant je pourrais vous le dire vous le répéter et quoi encore et pourquoi je ne prends rien pour acquis je veux qu'on m'explique je veux comprendre et par le fait même prendre vos routes et vos détours pour aller y voir de plus près je pourrais vous parler malheur vous parler chagrin parler bonheur

Je pourrais continuer longtemps prévoir ma mort dire dans un ultime secret enfin délivré de sa coquille d'huître que je mourrai jeune très jeune il est impossible de mourir vieux 
je n'aurai donc que 89 ans je serai là dans un champ sauvage comme je les aimais tant étant enfant

je serai là à genoux penché sur une talle de bleuets entouré d'épilobes de marguerites de jargeaux de crêtes-de-coq de mélinots blancs de chicorées bleues de trèfles de chardons je serai là penché comme en prière

il y aura des oiseaux

et tout à coup mon coeur s'arrêtera complètement de battre

je me lèverai porterai la main à ma poitrine

et je dirai tiens enfin voilà c'est fait

ce n'était pas compliqué j'ai vécu

définitivement peut-être j'ai vécu allons donc voir

était-ce bon ou bien ou finement fin

et trop subtil pour les mots

Mais pourquoi toujours chercher à dire à vous le dire je ne veux point m'entêter je ne cherche pas à vous convaincre de l'évidence de mes dires qui viennent au hasard de moi de mes regards attentifs aux coeurs

Moi je n'ai rien à dire peut-être comme vous qui faites je crois un peu le même chemin parfois évidemment inéluctablement je vous ai déjà rencontrés je vous ai déjà rencontrés mais où mais où déjà

Je parle je parle

moi qui n'avais rien à dire

tout à fait au hasard je parle et parle et parle

c'est toujours comme ça

C'est toujours comme ça!!! 